\title{
Amorolfine Hydrochloride
}

National Cancer Institute

\section{Source}

National Cancer Institute. Amorolfine Hydrochloride. NCI Thesaurus. Code C81507.

The hyrochloride salt form of amorolfine, a morpholine antifung al agent. Amorolfine inhibits delta-14-reductase and delta-7,8-isomerase, which depletes ergosterol and causes ignosterol to accumulate in the fungal cytoplasmic cell membrane. 